\title{
Abelian Dominance in Wilson Loops
}

\author{
Y. M. Cho \\ Asia Pacific Center for Theoretical Physics, \\ and \\ Department of Physics, College of Natural Sciences, Seoul National University, Seoul 151-742 \\ Korea \\ ymcho@yongmin.snu.ac.kr
}

\begin{abstract}
It has been conjectured that the Abelian projection of QCD is responsible for the confinement of color. Using a gauge independent definition of the Abelian projection which does not employ any gauge fixing, we provide a strong evidence for the Abelian dominance in Wilson loop integral. In specific we prove that the gauge potential which contributes to the Wilson loop integral is precisely the one restricted by the Abelian projection.
\end{abstract}

PACS numbers: 12.38.Aw, 12.38.Lg, 11.15.-q, 11.15.Ha

The confinement problem in QCD is perhaps one of the most difficult problems in theoretical physics. It has long been argued that the monopole condensation could provide the confinement of the color through a dual Meissner effect [1,2]. More explicitly it has been conjectured that the restricted part of QCD which comes from the "Abelian projection" of the theory to its maximal Abelian subgroup is responsible for the dynamics of the dual Meissner effect [2,3]. This conjecture, which asserts that in non-Abelian gauge theory only the degrees which correspond to its maximal Abelian subgroup should play the important role in the infra-red limit of the theory, is generally known as the "Abelian dominance", and has recently been readdressed by many authors in the literature [4.5]. But the actual proof of the Abelian dominance and the monopole condensation in the low energy limit of QCD has remained difficult.

A simple criterion for the confinement is given by the Wilson loop: the "area law" for the vacuum expectation value of the Wilson loop for a large current loop of a colored source, if established, could assure the confinement. This suggests that the Abelian dominance could be tested through the Wilson loop calculation. In this direction, a remarkable progress has been made by the numerical simulation during the last decade. In fact, the lattice calculation has confirmed the Abelian dominance, and showed that the dominant contribution to the string tension of the $q \bar{q}$ pair (about $92 \%$ ) comes from the Abelian projection of the theory [6, 7 . If If this is so, one should be able to prove the Abelian dominance with a field-theoretic method, independent of the numerical simulation. The purpose of this Letter is to provide a theoretical proof of the Abelian dominance in the Wilson loop integral. In specific we prove that it is the restricted connection, more precisely the gauge invariant part of the restricted connection, which contributes to the Wilson loop integral. Furthermore we show that the vacuum expectation value of the Wilson loop integral can be expressed as the weighted average of the loop integral of the restricted connection, weighted by the effective action of the restricted $Q C D$. This, together with the proof of the monopole condensation in the restricted QCD, will endorse the dual Meissner effect as the dynamical mechanism for the confinement of color in QCD.

Consider a non-Abelian gauge theory of a given gauge group $G$,

$$
\mathcal{L}=-\frac{1}{4} \vec{F}_{\mu \nu}^{2}
$$

where $\vec{F}_{\mu \nu}$ is the field strength. To prove the Abelian dominance in the infra-red limit, one must first clarify the meaning of the Abelian projection, and show how to project out the connection which corresponds to the maximal Abelian subgroup $H$ from the full non-Abelian connection of the group G. For $S U(2)$ this means that one should tell how to separate the $U(1)$ component of the connection in a gauge-independent way. To do this, one must select the $U(1)$ direction at each space-time point, and make a gauge-independent projection of the connection which contains only the $U(1)$ degree. This can be done by introducing a unit iso-triplet scalar field $\hat{n}(x)$ which transforms covariantly under the gauge transformation, and insisting that $\hat{n}$ remains unchanged under the parallel transport [2]. So we require $\hat{n}$ to be a covariant constant,

$$
D_{\mu} \hat{n}=0 \quad\left(\hat{n}^{2}=1\right) .
$$

Clearly $\hat{n}$ selects the $U(1)$ direction at each space-time point, and the parallel transport (2) provides the desired Abelian projection of the full connection $\vec{A}_{\mu}$,

$$
\overrightarrow{A_{\mu}} \longrightarrow \hat{A}_{\mu}=A_{\mu} \hat{n}-\frac{1}{g} \hat{n} \times \partial_{\mu} \hat{n},
$$

where $A_{\mu}=\hat{n} \cdot \vec{A}_{\mu}$ is the "naive" Abelian component (the electric potential) of the connection. This $\hat{A}_{\mu}$ is the restricted connection we introduced some time ago 
[2, 3]. It has many interesting features. First, $\hat{n}$ being gauge covariant, the projection (3) is obviously gaugeindependent. Moreover, $\hat{A}_{\mu}$ retains the full $S U(2)$ gauge degrees of freedom even though it is clearly restricted. This is because the gauge-independent projection still makes it an $S U(2)$ connection. Indeed, under an arbitrary gauge transformation specified by an infinitesimal parameter $\vec{\theta}$, one has

$$
\delta \hat{n}=-\vec{\theta} \times \hat{n}, \delta \vec{A}_{\mu}=\frac{1}{g} D_{\mu} \vec{\theta},
$$

which guarantees

$$
\delta \hat{A}_{\mu}=\frac{1}{g} \hat{D}_{\mu} \vec{\theta}=\frac{1}{g}\left(\partial_{\mu} \vec{\theta}+g \hat{A}_{\mu} \times \vec{\theta}\right) .
$$

More importantly, $\hat{A}_{\mu}$ retains the full topological characteristics of the original non-Abelian potential. In fact, the isolated singularities of $\hat{n}$ defines $\pi_{2}\left(S^{2}\right)$ which describes the non-Abelian monopoles [2,8]. Indeed $\hat{A}_{\mu}$ with $A_{\mu}=0$ and $\hat{n}=\hat{r}$ describes precisely the $\mathrm{Wu}$-Yang monopole [9]. Besides, with the $S^{3}$ compactification of $R^{3}, \hat{n}$ defines the Hopf invariant $\pi_{3}\left(S^{2}\right)$ which describes the topologically distinct vacuua [10].

The above discussion implies that there exists a subclass of the non-Abelian gauge theory, the restricted gauge theory, which contains only the restricted potential which nevertheless has the full non-Abelian gauge degrees of freedom [2,3]. To understand this, notice that with the restricted connection (3) one has

$$
\begin{gathered}
\hat{F}_{\mu \nu}=\left(F_{\mu \nu}+H_{\mu \nu}\right) \hat{n} \\
F_{\mu \nu}=\partial_{\mu} A_{\nu}-\partial_{\nu} A_{\mu} \\
H_{\mu \nu}=-\frac{1}{g} \hat{n} \cdot\left(\partial_{\mu} \hat{n} \times \partial_{\nu} \hat{n}\right)=\partial_{\mu} \tilde{C}_{\nu}-\partial_{\nu} \tilde{C}_{\mu},
\end{gathered}
$$

where $\tilde{C}_{\mu}$ is the monopole potential. To find an explicit form of $\tilde{C}_{\mu}$ let

$$
S=\exp \left(-t_{3} \gamma\right) \exp \left(-t_{2} \alpha\right) \exp \left(-t_{3} \beta\right)
$$

where $t_{i}$ are the adjoint representation of the $S U(2)$ generators, and let

$$
\hat{n}_{i}=S^{-1} \hat{e}_{i} \quad(i=1,2,3)
$$

where $\hat{e}_{1}=(1,0,0), \hat{e}_{2}=(0,1,0)$, and $\hat{e}_{3}=(0,0,1)$. Now we identify $\hat{n}$ to be $\hat{n}_{3}$,

$$
\hat{n}=\hat{n}_{3}=(\sin \alpha \cos \beta, \sin \alpha \sin \beta, \cos \alpha) .
$$

Then under the gauge transformation $S$, one has

$$
\begin{gathered}
\hat{A}_{\mu} \longrightarrow\left(A_{\mu}+\tilde{C}_{\mu}\right) \hat{e}_{3}, \\
\hat{F}_{\mu \nu} \longrightarrow\left(F_{\mu \nu}+H_{\mu \nu}\right) \hat{e}_{3},
\end{gathered}
$$

where

$$
\begin{aligned}
\tilde{C}_{\mu} & =\frac{1}{g}\left(\cos \alpha \partial_{\mu} \beta+\partial_{\mu} \gamma\right) \\
& =-\frac{1}{g} \hat{n}_{1} \cdot \partial_{\mu} \hat{n}_{2} .
\end{aligned}
$$

This shows that $\tilde{C}_{\mu}$ describes precisely the Dirac's monopole potential around the isolated singularities of $\hat{n}$. So the restricted gauge theory describes the dual dynamics of the color charge and the non-Abelian monopole.

With the restricted connection it is easy to obtain the full connection $\vec{A}_{\mu}$. Since the connection space forms an affine space, one can obtain a most general non-Abelian connection $\vec{A}_{\mu}$ simply by adding to $\hat{A}_{\mu}$ a gauge-covariant vector field $\vec{X}_{\mu}$ which is orthogonal to $\hat{n}$,

$$
\begin{aligned}
\vec{A}_{\mu} & =A_{\mu} \hat{n}-\frac{1}{g} \hat{n} \times \partial_{\mu} \hat{n}+\vec{X}_{\mu} \\
& =\hat{A}_{\mu}+\vec{X}_{\mu} .
\end{aligned}
$$

From (4) and (5) one can easily confirm that $\vec{X}_{\mu}$ indeed forms a covariant multiplet,

$$
\delta \vec{X}_{\mu}=-\vec{\theta} \times \vec{X}_{\mu}
$$

With (11) one has

$$
\vec{F}_{\mu \nu}=\left(F_{\mu \nu}+H_{\mu \nu}+X_{\mu \nu}\right) \hat{n}+\hat{D}_{\mu} \vec{X}_{\nu}-\hat{D}_{\nu} \vec{X}_{\mu}
$$

where $X_{\mu \nu}=g \hat{n} \cdot\left(\vec{X}_{\mu} \times \vec{X}_{\nu}\right)$. Furthermore with

$$
\vec{X}_{\mu}=X_{\mu}^{1} \hat{n}_{1}+X_{\mu}^{2} \hat{n}_{2}
$$

one has

$$
\begin{aligned}
\hat{D}_{\mu} \vec{X}_{\nu} & =\left[\partial_{\mu} X_{\nu}^{1}-g\left(A_{\mu}+\tilde{C}_{\mu}\right) X_{\nu}^{2}\right] \hat{n}_{1} \\
& +\left[\partial_{\mu} X_{\nu}^{2}+g\left(A_{\mu}+\tilde{C}_{\mu}\right) X_{\nu}^{1}\right] \hat{n}_{2}
\end{aligned}
$$

So one could express the Lagrangian explicitly in terms of $A_{\mu}, \hat{n}$, and $\vec{X}_{\mu}$,

$$
\begin{aligned}
\mathcal{L}= & -\frac{1}{4}\left(F_{\mu \nu}+H_{\mu \nu}+X_{\mu \nu}\right)^{2} \\
& -\frac{1}{4}\left(\hat{D}_{\mu} \vec{X}_{\nu}-\hat{D}_{\nu} \vec{X}_{\mu}\right)^{2}
\end{aligned}
$$

Clearly this describes a $U(1)$ gauge theory coupled to a charged vector field $\vec{X}_{\mu}$. But the important point here is that the $U(1)$ potential is given by $A_{\mu}+\tilde{C}_{\mu}$, not just $A_{\mu}$. The corresponding equations of motion is given by

$$
\begin{aligned}
\partial_{\mu}\left(F_{\mu \nu}+H_{\mu \nu}+X_{\mu \nu}\right) & =-g \hat{n} \cdot \vec{X}_{\mu} \times\left(\hat{D}_{\mu} \vec{X}_{\nu}-\hat{D}_{\nu} \vec{X}_{\mu}\right) \\
\hat{D}_{\mu}\left(\hat{D}_{\mu} \vec{X}_{\nu}-\hat{D}_{\nu} \vec{X}_{\mu}\right) & =g\left(F_{\mu \nu}+H_{\mu \nu}\right. \\
& \left.+X_{\mu \nu}\right) \hat{n} \times \hat{X}_{\mu}
\end{aligned}
$$

This allows us to interpret $\vec{X}_{\mu}$ as "the valence gluon" which plays the role of a colored source of the restricted theory [2]3]. More importantly this implies that $\vec{X}_{\mu}$, just 
like the quarks, represents simply another colored source which has to be confined itself. This is the reason why only the restricted connection (3) should play the dominant role in the Wilson loop integral.

Notice that under the inverse gauge transformation $S^{-1}$ which rotates $\hat{e}_{3}$ to $\hat{n}$, one must have

$$
\begin{aligned}
\hat{A}_{\mu} & =\left(A_{\mu}+\tilde{C}_{\mu}\right) \hat{n}+\frac{1}{g} \operatorname{tr}\left(-\frac{1}{2} \vec{t} S^{-1} \partial_{\mu} S\right) \\
& =A_{\mu} \hat{n}-\frac{1}{g} \hat{n} \times \partial_{\mu} \hat{n} .
\end{aligned}
$$

More importantly under the gauge transformation (3) one has

$$
\begin{aligned}
\delta\left(A_{\mu}+\tilde{C}_{\mu}\right) & =\delta\left(\hat{n} \cdot \vec{A}_{\mu}\right)-\frac{1}{g} \delta\left(\hat{n}_{1} \cdot \partial_{\mu} \hat{n}_{2}\right) \\
& =0 .
\end{aligned}
$$

This shows that $\left(A_{\mu}+\tilde{C}_{\mu}\right) \hat{n}$ is the gauge covariant part of the restricted connection. This in turn allows us to identify the gauge covariant part $\vec{A}_{\mu}^{(c)}$ of the full connection,

$$
\begin{aligned}
\vec{A}_{\mu} & =\left(A_{\mu}+\tilde{C}_{\mu}\right) \hat{n}+\vec{X}_{\mu}+\frac{1}{g} \operatorname{tr}\left(-\frac{1}{2} \vec{t}^{-1} \partial_{\mu} S\right) \\
& =\vec{A}_{\mu}^{(c)}+\frac{1}{g} \operatorname{tr}\left(-\frac{1}{2} \vec{t}^{-1} \partial_{\mu} S\right) .
\end{aligned}
$$

This observation will become important in the Wilson loop calculation.

Now we are ready to discuss Wilson loop integral along a closed curve $C$. The integral, although conceptionally simple, has remained very difficult to carry out. But an important step to simplify the integral was made by Diakonov and Petrov [11, who showed that integral for an arbitrary representation $T_{i}$ can be expressed as a functional integral over all gauge transformations $S(t)$ along the loop,

$$
\begin{aligned}
W(C)= & \operatorname{tr} P \exp \left[-\oint A_{\mu}^{i} T_{i} d x^{\mu}\right] \\
= & \int \mathcal{D} S(t) \exp \left[i \frac { T } { 2 } \oint \operatorname { t r t } { } _ { 3 } \left(S \mathcal{A}_{\mu} S^{-1}\right.\right. \\
& \left.\left.+\frac{1}{g} S \partial_{\mu} S^{-1}\right) d x^{\mu}\right],
\end{aligned}
$$

where $\mathcal{A}_{\mu}=\vec{A}_{\mu} \cdot \vec{t}$ and $T$ is the Casimir invariant (i.e., the color charge) of the representation $T_{i}$. Now from (19) we have

$$
\begin{aligned}
-\frac{1}{2} \operatorname{tr} & {\left[t_{3}\left(S \mathcal{A}_{\mu} S^{-1}+\frac{1}{g} S \partial_{\mu} S^{-1}\right)\right] } \\
& =-\frac{1}{2} \operatorname{tr}\left[S^{-1} t_{3} S\left(\mathcal{A}_{\mu}-\frac{1}{g} S^{-1} \partial_{\mu} S\right)\right] \\
& =A_{\mu}+\tilde{C}_{\mu}
\end{aligned}
$$

Notice that this is precisely the gauge invariant part of the restricted potential

$$
A_{\mu}+\tilde{C}_{\mu}=\hat{n} \cdot \vec{A}_{\mu}^{(c)} .
$$

This makes us to identify the gauge field configuration which is relevant to the Wilson loop integral. It is indeed the restricted gauge potential, more precisely the gauge invariant part of the restricted potential, that contributes to the Wilson loop integral. This strongly endorses the Abelian dominance in QCD [2,3].

With (20) and (21) we can now obtain the desired expression for the vacuum expectation value of the Wilson loop,

$$
\begin{aligned}
<W(C)>= & \int \mathcal{D} A_{\mu} \mathcal{D} \hat{n} \mathcal{D} \vec{X}_{\mu} \exp \left[-\frac{1}{4} \int \vec{F}_{\mu \nu}^{2} d^{4} x\right. \\
& \left.-i T \oint\left(A_{\mu}+\tilde{C}_{\mu}\right) d x^{\mu}\right]
\end{aligned}
$$

By integrating out the $\vec{X}_{\mu}$ degrees of freedom with a proper gauge fixing, one can express the integral as the vacuum average of (20) over the effective Lagrangian $\hat{\mathcal{L}}_{\text {eff }}$ of the restricted $\mathrm{QCD}$,

$$
\begin{aligned}
<W(C)>= & \mathcal{D} A_{\mu} \mathcal{D} \hat{n} \exp \left[-\int \hat{\mathcal{L}}_{\text {eff }} d^{4} x\right. \\
& \left.-i T \oint\left(A_{\mu}+\tilde{C}_{\mu}\right) d x^{\mu}\right] \\
= & \mathcal{D} A_{\mu} \mathcal{D} \tilde{C}_{\mu} \exp \left\{-\int\left[\hat{\mathcal{L}}_{\text {eff }}\right.\right. \\
& \left.\left.+i T\left(A_{\mu}+\tilde{C}_{\mu}\right) j^{\mu}\right] d^{4} x\right\}
\end{aligned}
$$

where

$$
j^{\mu}=\int \delta^{4}(x-z(t)) \frac{d z^{\mu}}{d t} d t,
$$

and we have changed the variable $\hat{n}$ by $\tilde{C}_{\mu}$ (it is understood that $\mathcal{D} \tilde{C}_{\mu}$ includes the Jacobian for the change of variable). Notice that here it is the effective action, not the bare action, of the restricted theory which appeares in the loop integral. This effective action will contain all the dynamical informations necessary for the confinement.

The above result shows that one can reduce the evaluation of the Wilson loop integral to the evaluation of the generating functional of the restricted gauge theory for the gauge invariant external current $j_{\mu}$. This is really remarkable, but perhaps not so surprising. It has been known that the evaluation of the Wilson loop integral could be related to the evaluation of a gauge invariant part of the generating functional which is invariant under the gauge transformation of the external current $\vec{j}_{\mu}[1]$. Our result not only confirms this but more importantly drastically simplifies the integral, which is made possible by identifying the precise field configuration which contributes to the Wilson loop.

To proceed further one must know the effective action of the restricted QCD. The actual calculation of the effective action goes beyond the scope of this paper, but 
fortunately one can do this at one loop level. With the monopole potential as the classical background, one can show that the effective action does indeed provide the monopole condensation [12]. With this the physics behind our main result (24) becomes unmistakable. The monopole condensation should squeeze the electric flux of the colored source into a string, and generate the confining potential for the colored objects.

The main point of the paper is to express the nonAbelian Wilson loop integral in terms of the restricted potential. Mathematically this amounts to reducing the non-Abelian Stoke's integral to a quasi-Abelian Stoke's integral. There have been many unsuccessful attempts to do this in the literature. We have shown how to do this with the Abelian projection. This by itself does not prove the confinement. The actual proof of the confinement should come from the calculation of the effective action of the restricted QCD [12].

We close with the following remarks:

1) It must be emphasized that there is a crucial difference between our definition of the Abelian projection and that of the others. In the popular definition of ' $t$ Hooft, the Abelian projection is regarded as a partial gauge fixing (called the maximal Abelian gauge) of $G / H$ degrees 四河. In another definition which does not employ any gauge fixing, the projection is not supposed to depend on any particular set of field configurations [13]. In comparison our Abelian projection (3) selects a particular set of field configurations (the restricted potential), and is explicitly gauge independent. Furthermore after the projection the restricted potential still enjoys the full non-Abelian gauge degrees of freedom.

2) One should keep in mind that one does not have to have the linear potential, and the Wilson loop integral does not have to generate the area law, to guarantee the confinement. This is because asymtotically the $q \bar{q}$ string can break by creating the pairs. This "screening effect" could saturate the linear potential, and thus compromize the area law. Only when one neglects this dynamical possibility of the pair creation in the Wilson loop integral, one can obtain the area law.

3) The lattice simulations suggest that the gluon loop does not generate a linear potential, so that the area law may not hold for the adjoint representations [14]. This has been (incorrectly) attributed to the screening effect in the literature. This appears to contradict with our result, according to which the quark and the gluon loops give the same integral expression except for the color charge $T$. But this appearence is misleading, because they contribute oppositely to the effective action. In fact one can demonstrate that, just as in the asymtotic freedom, the gluons tend to make pair annihilations but the quarks tend to make pair creations. This means that the gluons generate an "anti-screening effect", while the quarks generate the screening effect [12]. So our result can naturally explain why the gluon loop does not obey the area law. But we emphasize that the correct reason for this is not the screening effect, but the anti-screening effect.

It must be straightforward to generalize our result to an arbitrary group [3,8]. A more detailed discussion, including the proof of the monopole condensation and the derivation of the effective action for the restricted QCD, will be given in a forthcoming paper [12, 15].

The author thanks Professor C. N. Yang for fruitful discussions and encouragements, and appreciates interesting discussions with D. Diakonov, L. Faddeev, K. Fujikawa, A. Niemi, and H. Toki. The work is supported in part by Korean Science and Engineering Foundation, and by the BK21 project of Ministry of Education.

[1] Y. Nambu, Phys. Rev. D10, 4262 (1974); S. Mandelstam, Phys. Rep. 23C, 245 (1976); G. 't Hooft, Nucl. Phys. B190, 455 (1981).

[2] Y. M. Cho, Phys. Rev. D21, 1080 (1980).

[3] Y. M. Cho, Phys. Rev. Lett. 46, 302 (1981); Phys. Rev. D23, 2415 (1981).

[4] Z. Ezawa and A. Iwazaki, Phys. Rev. D25, 2681 (1982); T. Suzuki, Prog. Theor. Phys. 80, 929 (1988); H. Sugamura, S. Sasaki, and H. Toki, Nucl. Phys. B435, 207 (1995); K. Kondo, Phys. Rev. D57, 7467 (1998); D58, 105016 (1998).

[5] R. Brower, K. Orginos, and C. I. Tan, Phys. Rev. D55, 6313 (1997); L. Quandt and H. Reinhardt, Int. J. Mod. Phys. A13, 4049 (1998); L. Faddeev and A. Niemi, Phys. Rev. Lett. 82, 624 (1999); Phys. Lett. B449, 214 (1999).

[6] A. Kronfeld, G. Schierholz, and U. Wiese, Nucl. Phys. B293, 461 (1987); T. Suzuki and I. Yotsuyanagi, Phys. Rev. D42, 4257 (1990).

[7] J. Stack, S. Neiman, and R. Wensley, Phys. Rev. D50, 3399 (1994); G. Bali, V. Bornyakov, M. Müller-Preussker, and K. Schilling, Phys. Rev. D54, 2863 (1996).

[8] Y. M. Cho, Phys. Rev. Lett. 44, 1115 (1980); Phys. Lett. B115, 125 (1982).

[9] T. T. Wu and C. N. Yang, Phys. Rev. D12, 3845 (1975).

[10] Y. M. Cho, Phys. Lett. B81, 25 (1979).

[11] D. Diakonov and V. Petrov, Phys. Lett. B224, 131 (1989); B242, 425 (1990); M. Faber, A. Ivanov, N. Troitskaya, and M. Zach, hep-th/9907048.

[12] Y. M. Cho and D. G. Pak, hep-th/9906198, hepth/0006051, in Proceedings of TMU-Yale Symposium on Dynamics of Gauge Fields, edited by T. Appelquest and H. Minakata (Universal Academy Press, Tokyo) 1999.

[13] M. Faber, J. Greensite, S. Olejnik, Phys. Rev. D57, 2603 (1998); M. Ogilvie, Phys. Rev. D59, 74505 (1999).

[14] C. Michael, Nucl. Phys. B26, 417 (1992); J. Ambjorn, J. Giedt, and J. Greensite, hep-lat/9907021.

[15] Y. M. Cho and D. G. Pak, to be published. 